\title{
SECURE DATA GROUP SHARING AND CONDITIONAL DISSEMINATION WITH MULTI-OWNER IN CLOUD COMPUTING
}

\author{
Sai Navya G \\ MCA Scholar, School of CS \& IT, Dept. of MCA, Jain (Deemed-to-be) University, Bangalore
}

Article DOI: $\underline{\text { https://doi.org/10.36713/epra5768 }}$

\begin{abstract}
Secure inquiry methods cloud information permit an approved client to question information records of interest by submitting encoded question catchphrases to the cloud worker in a protection safeguarding way. In any case, practically speaking, the returned question results might be off base or deficient in the deceptive cloud climate. For instance, the cloud worker may interntionally exclude some certified outcomes to spare computational assets and correspondence overhead. Along these lines, a well-working secure inquiry framework ought to give a question results check instrument that permits the information client to confirm results. In this paper, we plan a protected, effectively coordinated, and fine-grained inquiry results confirmation system, by which, given a scrambled question results set, the inquiry client not exclusively can confirm the accuracy of every information document in the set yet additionally can additionally check the number of or which qualified information records in the set is deficient before unscrambling. The sheme is free coupling to concrete secure inquiry strategies and can be effectively integreated into any protected question plot. We accomplish the objective by building secure confirmation object for scrambled cloud information. Execution assessment shows that the proposed plans are useful and effective.
\end{abstract}

\section{I.INTRODUCTION}

Distributed computing is the utilization of registering assets that are conveyed as an assistance over an organization. The name comes from the regular utilization of a cloud formed image as a reflection for the perplexing framework it contains in framework graphs. Distributed computing depends far off administrations with a client information, programming and calculation.

The objective of distributed computing is to apply conventional supercomputing or superior registering power, ordinarily utilized by military and exploration offices, this distributed computing utilizes organizations of enormous gatherings of workers regularly running minimal effort buyer PC innovation with specific associations with spread information preparing errands across them. This mutual IT framework contains enormous pools of frameworks that are together.

As of late, with the developing prevalence of cloud computing,how to safely and productively search over encoded cloud information turns into an exploration center. A few methodologies have been proposed dependent on customary accessible encryption plans, which mean to ensure information security and question protective measures with better inquiry productive for distributed computing. Wang et al. applied hash clain strategy to actualize the culmination check of question results by inserting the scrambled confirmation data into their proposed secure accessible record. Sun et al. utilized scrambled list tree structure to execute secure question results check usefulness. In this plan, when the question closes, the cloud worker returns inquiry results alongside a base scrambled file tree, at that point the information client look through this base list tree utilizing a similar pursuit calculation an obvious secure inquiry conspire over encoded cloud information dependent on quality based encryption method in the public-key setting.

In this paper, we stretch out and fortify our work to make it more pertinent in the cloud climate and safer to against unscrupulous cloud worker. The fundamental commitments of this paper are we officially propose the undeniable secure framework model and danger model and plan a fine grained inquiry results confirmations conspire for secure over encoded cloud information. We proposed a short signature strategy dependent on 


\section{EPRA International Journal of Research and Development (IJRD)

\author{
Volume: 5 | Issue: 11 | November 2020
} \\ - Peer Reviewed Journal}

certificateless public-key cryptography to ensure the genuineness of the check objects themselves. We plan a novel confirmation object demand strategy dependent on paillier encryption, where the cloud worker thinks nothing about what the information client is mentioning for and which check objects are gotten back to the client.

We give the proper security definition and confirmation and lead broad execution investigations to assess the precision and productivity of our proposed plans. Our plan can check the rightness of each encoded inquiry result or further precisely discover the number of or which qualified information documents are returned by the exploitative cloud worker. A short signature strategy is intended to ensure the genuineness of confirmation object iteself

\section{II . LITERATURE SURVEY}

\section{Creators: K.Ren:}

Distributed computing speaks to the present most energizing figuring change in perspective in data innovation. Nonetheless, security and protection are seen as essential obstructions to its wide reception. Here, the creators diagram a few basic security challenges and rouse further examination of security answers for reliable public cloud climate.

Creators: S.Kamara and K.Lauter:

We consider the issue of building a safe distributed storage administration on top of a public cloud foundation where the specialist organization isn't totally trusted by the client. We portray, at a significant level, a few design that consolidate later and non-standard cryptographic natives to accomplish our goal.we study the advantages such an engineering would give to the two clients and specialist organizations and give a review of ongoing advances in cryptography roused explicitly by distributed storage.

Creators: D.Song, D.Wagner:

It is attractive to store information on information stockpiling workers, for example, mail workers and fike workers in encoded structure to decrease security and protection hazards yet this normally suggests that one needs to forfeit usefulness for security realized how to let the information stockpiling worker play out the inquiry and answer the words, it was not recently realized how to let the information stockpiling worker play out the hunt and answer the question, without loss of information secrecy. We portray our cryptographic plans for the issue of looking on scrambled information and give confirmations of security to the subsequent crypto frameworks. Our methods have various critical preferences. They are provably secure: they give provably safely to encryption, as in the untrusted worker can't master anything about the plaintext when just given the ciphertext; they give inquiry disengagement to look, implying that the untrusted worker can't pick up much else about the plaintext than the query item; they give controlled looking, so that the untrusted worker can't look for a subjective word without the client's authorization;they additionally uphold covered up queries,so that the client may approach the untrusted worker to look for a mystery word without uncovering the word to the worker. The calculations introduced are straightforward, quick

\section{SYSTEM ARCHITECTURE}

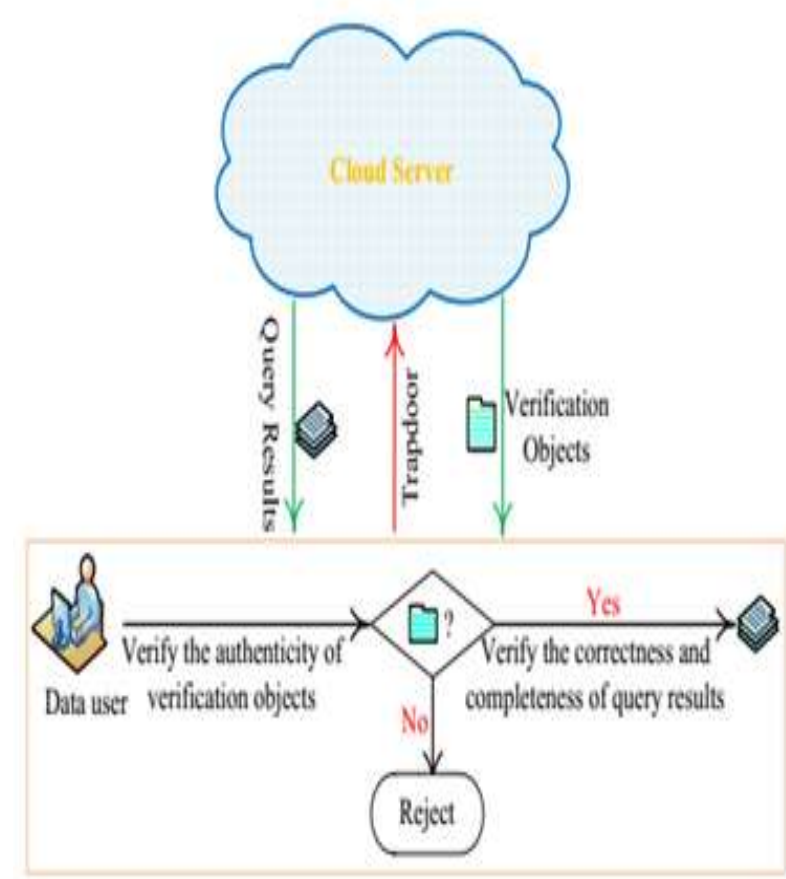

\section{DATA FLOW DIAGRAM}

The DFD is additionally called bubble chart. It is a straightforward graphical formalism that can be utilized to speak to a framework as far as info information to the framework, different handling did on this information, and the yield information is created by this framework. The information stream outline is one od the main displaying instruments. It is utilized to demonstrate the framework segments. These parts are the framework cycle, the information utilized by the cycle, an outside element that associates with the framework and the data streams in the framework. DFD shows how the data travels through the framework and how it is altered by a progression of changes. It is a graphical procedure that portrays data stream and the changes that are applied as information moves from contribution to yield. DFD is otherwise called bubble diagram. A DED be utilized to speak to a framework at any degree of reflection. DFD might be parceled into level that speak to expanding data stream and utilitarian detail. 


\section{EPRA International Journal of Research and Development (IJRD)}

\section{Volume: 5 | Issue: 11 | November 2020}

- Peer Reviewed Journal

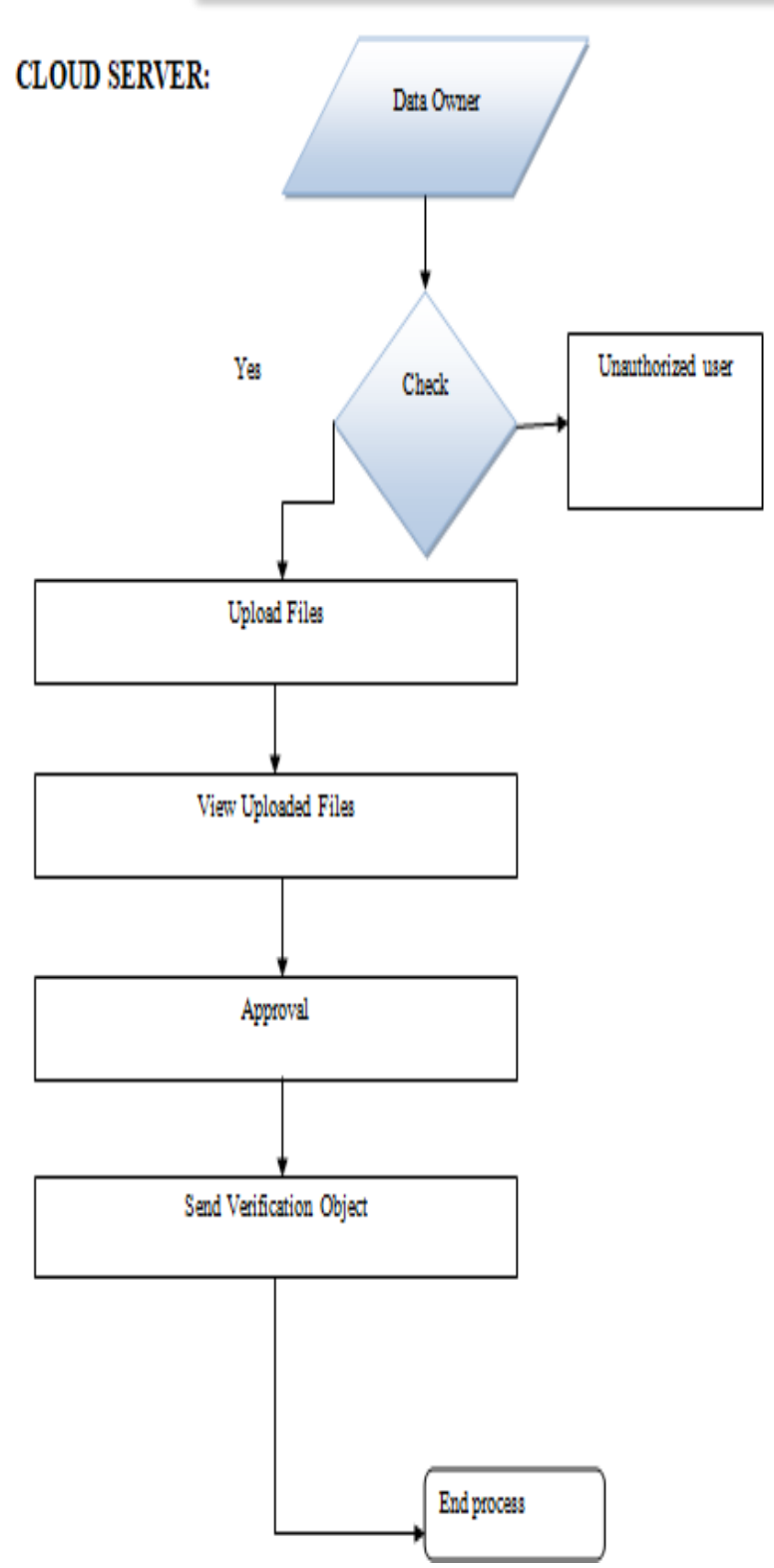

\section{V.GOALS}

The essential objectives in the UML are:

1. Give clients a prepared to utilize, expressive visual demonstrating language with the goal that they can create and trade significant models.

2. Give extendibility and specialixation components to expand the center ideas.

3. Be autonomous of specific programming language and advancement measure.

4. Give a proper premise to understanding the displaying language.

5. Energize the development of $\mathrm{OO}$ devices market.

6. Backing higher advancement ideas, for example, joint efforts, systems, examples and segments.

7. Incorporate prescribed procedures.

\section{CONCLUTION}

In this paper, we propose a safe, effortlessly incorporated, and fine grained inquiry results confirmation plot for secure hunt over encoded cloud information not quite the same as past works, our plan can check the rightness of each scrambled question result or further a precisely discover the number of or which qualified information records are returned by the unscrupulous cloud worker. Ashort signature strategy is intended to ensure the realness of check object itself. Also, we plan a protected check object demand procedure, by which the cloud worker thinks nothing about confirmation object is mentioned by the information client and really returned by the cloud worker. Execution and precision tests exhibit the legitimacy and proficiency of our proposed plot.

\section{REFERENCES}

1. P. Mell and T. Grance, "The nist definition of cloudcomputing, "http://dx.doi.org/10.602/NIST.SP.80 0-145.

2. S.Kamara and K.Lauter, "Cryptographic cloud storage," in Springer RLCPS, January 2010.

3. D.Song, D. Wanner, and A. Perrig, "Practical techniques for searches on encrypted data," in IEEE Symposiumon Security and Privacy,vol.8, 2000,pp.4455.

4. E.-J.Goh,"Secure indexes, "IACRe Print Cryptography Archieve, http://eprint.iacr.org/2003/213, Tech.Rep., 2003.

5. D.Boneh, G.D.Crescenzo, R.Oatrovsky, and G.Persiano, "Public-Key encryption with keyword search, "in EUROCRYPR,2004,pp.506-522.

6. R.Curtmola, J.Garay, S.Kamara, and R.Ostrovsky," Searchable symmetric encruption :im proved definition and efficient costructions, "in ACM CCS, vol. 19,2006,pp. 79-88.

7. M.Bellare, A.Boldyreva, and A.O'Neill, Deterministic and efficiently searchable encryption, "in Springer CRYPTO, 2007.

8. S.Kamara and C.Papamanthou, "Parallel and dynamic searchable symmetric encryption, in Financial Cryptography and Data Security, Springer Berlin Heidelberg,2013 ,pp.258-274.

9. M.Naveed, M.Prabhakaran, and C.a. Gunter,"Dynamic searchable encryption via blind storage," in IEEE S\&P, May 2014,pp.639-654.

10. C.Wang, N.Cao, J.Li, K.Ren, and W.Lou, "Secure ranked keyword search over encrypted cloud data, "in IEEE ICDCS,2010,pp. 253-262.

11. N.Cao, C.Wang, sM. Li, K. Ren, and W. Lou," Privacy-preserving multi-keyword ranked search over encrypted cloud data," in IEEE INFOCOM,2011, pp.829-837.

12. M. Bellare and P. Rogaway, Introduction to Modern Cryptography. Lecture Notes, 2001. 